\title{
Chapter 2 \\ Maintenance of Adult Stem Cells: Role of the Stem Cell Niche
}

\author{
Yoshiko Matsumoto, Hiroko Iwasaki, and Toshio Suda
}

\begin{abstract}
Self-renewing stem cells are maintained in functionally specific microenvironments that are termed niches. Optimum combination of intrinsic programs and extrinsic stimuli from niches is essential for maintaining stem cell quiescence and for proper mobilization and differentiation. The most advanced imaging technologies allow the clearer real-time visualization of the behavior of hematopoietic stem cells in living animal. Functions of niche factors, such as adhesion molecules and cytokines, are well studied. Concentration levels of local oxygen and reactive oxygen species also affect the fate of stem cells in their niches. Analysis of niche regulation will lead to a better understanding of stem cell behavior. Moreover, altering the cancer stem cell fate through modification of the niche regulation may be one of the candidates for new therapeutic strategies for diseases such as chemoresistant leukemia.
\end{abstract}

Keywords Hematopoietic stem cells - Endosteal niche - Perivascular niche

- Osteoblasts - Self-renewal • Symmetric division • Asymmetric division • Cell cycle $\bullet$ Reactive oxygen species
Abbreviations
Atm Ataxia telangiectasia mutated gene
BMT Bone marrow transplantation
BrdU 5-Bromo-2-deoxyuridine
CAR CXCL12-abundant reticular cells
Casr Calcium-sensing receptor
CDK Cyclin-dependent kinase
ECM Extracellular matrix

\footnotetext{
T. Suda $(\bowtie)$

Department of Cell Differentiation, Keio University School of Medicine, 35 Shinanomachi, Shinjuku-ku, Tokyo 160-8582, Japan

e-mail: sudato@sc.itc.keio.ac.jp
} 


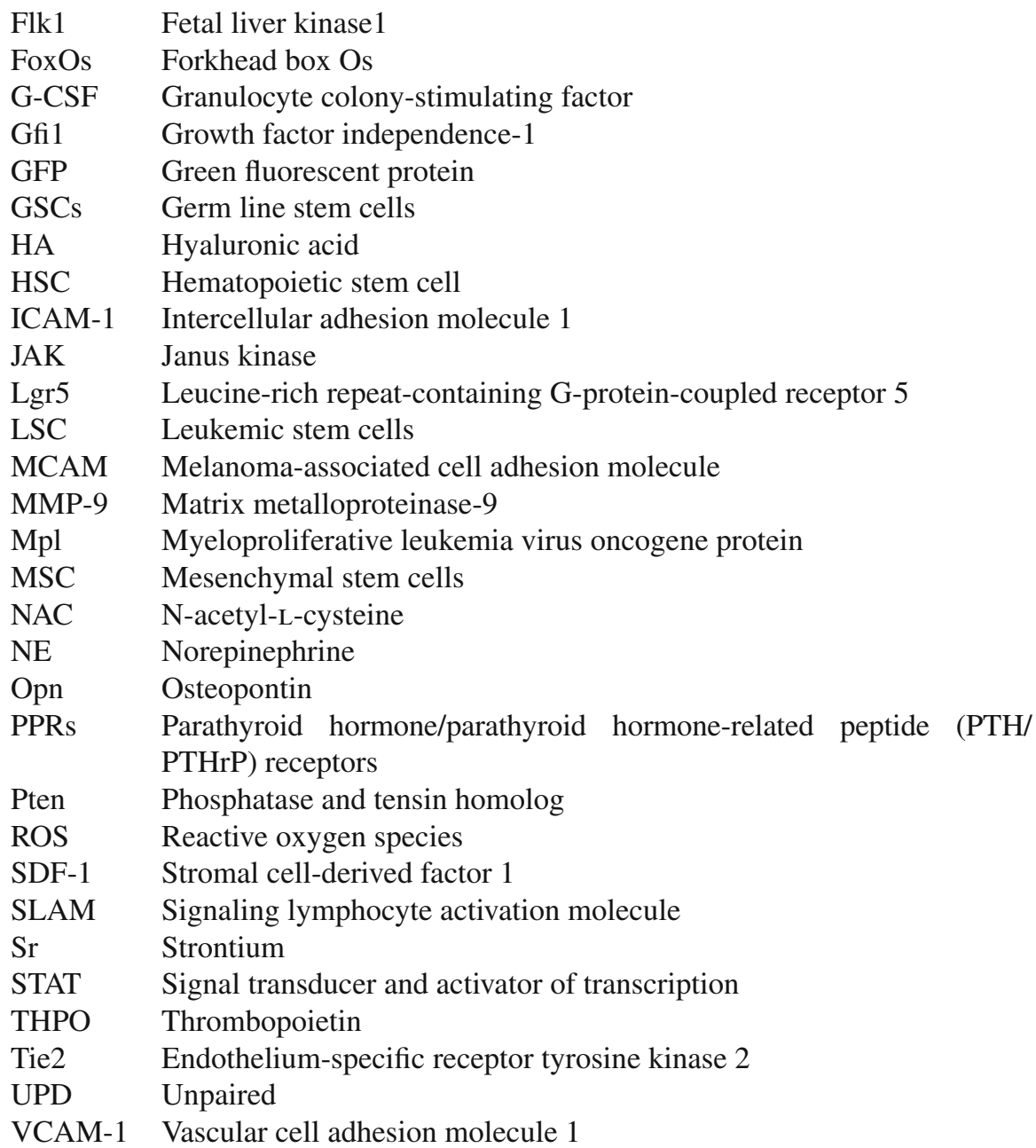

\subsection{Introduction}

Stem cells are the cells with two unique characteristics: self-renewal and differentiation into multiple lineages. They exist virtually in every organ and supply an adequate number of differentiated cells to maintain the functions of the organ. The existence of stem cells was first demonstrated in 1961 by Till and McCulloch in a study on hematopoiesis (Till and McCulloch 1961). The establishment of the concept of hematopoietic stem cells (HSCs) was followed by the discovery of tissue stem cells in other organs in mammals, e.g., epithelial stem cells, neural stem cells, and intestinal stem cells (Fuchs and Segre 2000). 
Stem cells can be found in specific areas of each organ termed "niches" where they maintain stem cell function. In 1978, Schofield proposed the concept of "niche" as the functionally specific microenvironment of the stem cells (Schofield 1978). This niche function was substantiated by the study of germ line stem cells (GSCs) in Drosophila, where the existence of niches in vivo was demonstrated for the first time (Xie and Spradling 2000). In female Drosophila, GSCs are found within the germarium, which is a generative region located at the tip of ovariole. Cap cells located at the tip of the germarium and adjacent to GSCs were first defined by Xie and Spradling as the key niche cells that support GSCs. Further studies revealed that GSCs are mainly and strictly governed by bone morphogenetic protein (BMP) signaling in the niche (Chen and McKearin 2003; Song et al. 2004). In contrast, apical hub cells act as a cellular niche in fly testes (Fuller and Spradling 2007).

In mammals, hematopoiesis has taken the lead in niche studies, while the variety of cells in organs and the complexity of tissues in mammals have hampered investigations into niche function.

\subsection{Quiescence and Self-Renewal}

In the mammalian hematopoietic system two types of stem cells have been identified: quiescent HSCs and cycling HSCs (Arai and Suda 2007). The quiescent state is necessary to maintain the self-renewal capacity of HSCs and to prevent stem cell exhaustion. Loss of p21, which is a G1 checkpoint regulator and a cyclin-dependent kinase (CDK) inhibitor, allows the escape of HSCs from quiescence and promotes their entrance into the cell cycle (Cheng et al. 2000). As a result, $p 21^{-1-}$ mice exhibit HSC proliferation and a consequent increase in the total number of HSCs, as well as an enhancement in their sensitivity to cell cycle-specific myelotoxic injury because of hematopoietic cell depletion. The self-renewal capacity of primitive cells was also impaired in serial bone marrow transplantation (BMT) of HSCs derived from $p 21^{-/-}$mice. HSCs lacking expression of growth factor independence-1 (Gfil), phosphatase and tensin homolog (Pten), or forkhead box Os (FoxOs) also exhibit activation of cell cycling and similar phenotypes (Hock et al. 2004a; Yilmaz et al. 2006; Zhang et al. 2006; Miyamoto et al. 2007; Tothova et al. 2007; Orford and Scadden 2008; Yalcin et al. 2008).

The cell cycle state is monitored mainly by detecting the retention of 5-bromo-2deoxyuridine (BrdU) in chromosomes. Bradford et al. reported that the BrdU is retained in primitive HSCs (Bradford et al. 1997). The Fuchs group developed a method using a histone $\mathrm{H} 2 \mathrm{~B} /$ green fluorescent protein (H2B-GFP), with which GFP-labeled, nondividing or slow-cycling cells can be sorted and collected (Tumbar et al. 2004). Using the BrdU and the H2B-GFP fusion protein, Trumpp and his colleague showed that the most primitive stem cells $\left(\mathrm{Lin}^{-} \mathrm{Sca}{ }^{+}{ }^{+} \mathrm{Kit}^{+} \mathrm{CD} 48^{-} \mathrm{CD} 150^{\mathrm{hi}} \mathrm{CD}\right.$ 34-HSCs) actually comprise two subsets of cells: a dormant HSC population, which most probably divides only every 145-193 days, and an activated HSC population, 
which divides every 28-36 days (Wilson et al. 2008). They proposed that the dormant population is activated to enter the cell cycle under conditions of hematopoietic stress to produce mature cells and then returns to quiescence after reestablishment of homeostasis, where they act as a silent reservoir during the normal state. Another group also used the H2B-GFP fusion protein to show that the $\mathrm{Lin}^{-} \mathrm{Scal}^{+} \mathrm{CKit}^{+}$ $\mathrm{CD} 150^{+} \mathrm{CD} 48^{-}$fraction, which is highly enriched for HSCs, represents a heterogeneous population in which less than $20 \%$ of cells divide at a slower rate (Foudi et al. 2009).

The maintenance of quiescent HSCs is the main role of the HSC niche in bone marrow (BM). Cytokines, growth factors, local environmental factors, and adhesion molecules that anchor HSCs to the endosteum play a key role in maintaining quiescence. The concept of coexistence of different types of HSCs in niches - quiescent and cycling stem cells - is now accepted and proved for other types of stem cells. For example, the Clevers group demonstrated that the leucine-rich repeat-containing G-protein-coupled receptor 5 (Lgr5)-positive cells in the pyloric gland are actively cycling pyloric stem cells, whereas villin ${ }^{+}$stem cells are quiescent (Qiao et al. 2007; Barker et al. 2010).

The ability to self-renew is the most important characteristic of stem cells. However, it is not recapitulated well in vivo and in vitro. The process of stem cell self-renewal differs from the maintenance of quiescence and has long been attributed to the capacity of stem cells to undergo symmetric and asymmetric cell division. The asymmetric division of a stem cell gives rise to both an exact copy of itself and a daughter cell that will differentiate into mature cells of the tissue. Asymmetric division of stem cells is supported by observation of GSCs in female Drosophilia (Deng and Lin 1997). When cell division occurs such that the mitotic spindle is oriented perpendicular to the niche, one daughter cell maintains its exposure to the niche and retains its stem cell properties while the other is displaced from it and undergoes differentiation (Yamashita et al. 2003). In this way, the commitment to differentiation of fly GSCs seems to be an "all-or-nothing" event. In contrast, this commitment process seems to be more complex in mammalian HSCs, in which both symmetric and asymmetric divisions are proposed to be present in the multistep models.

Stem cell fate is regulated by both intrinsic programs and extrinsic stimuli. For example, cells derived from a single progenitor and exposed to the same growth-promoting environment often develop into different kinds of colonies, indicating that fate decisions were controlled by intrinsic or stochastic mechanisms (Suda et al. 1984). Differential responsiveness of stem cells to extrinsic signaling rather than environmental variability (time and concentration) of stimuli also supports the intrinsic programming of stem cells. In contrast, the Schroeder group used time-lapse microscopy to show that the differentiation of progenitors of neutrophils and macrophages is regulated by external stimuli (Rieger et al. 2009). 
Interestingly, HSCs change their living space during ontology. In the midgestation embryo, the first definitive HSCs arise from the hemogenic endothelium on the ventral side of the aorta, enter the circulation, seed the placenta, and continue to expand and mature in the fetal liver. Before birth, cycling HSCs migrate from the liver to the developing BM (Orkin and Zon 2008). At the end of the preadult phase, organ growth slows down and eventually stops. Stem cell quiescence coincides with the switch from developmental expansion to homeostatic maintenance. Thus, it is important to distinguish adult hematopoiesis from the hematopoiesis that is associated with stem cell expansion in fetal liver and infantile BM. Quiescence is a characteristic feature of adult HSCs. In particular, slow cell cycling or quiescence confers stress resistance to adult stem cells (Fig. 2.1).

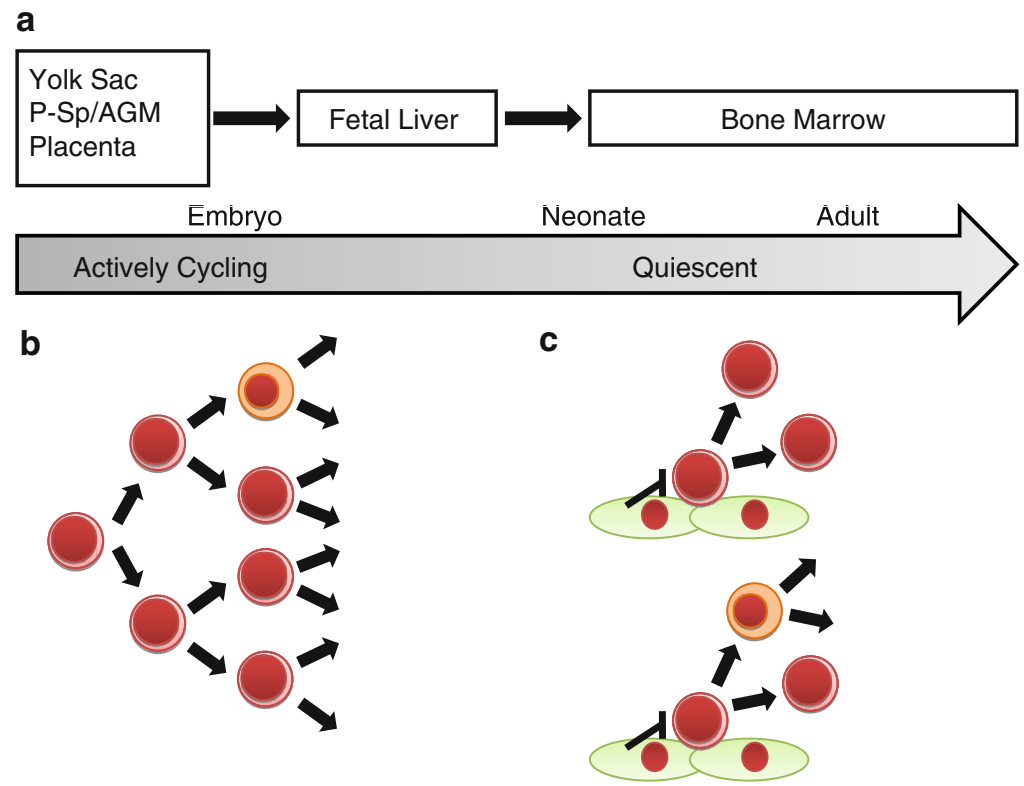

Fig. 2.1 A schema of mammalian hematopoiesis. (a) Primitive hematopoiesis occurs in the yolk sac where erythrocytes are mainly produced. The first definitive HSCs arise from the hemogenic endothelium on the ventral side of the aorta in the midgestation embryo, enter the circulation, seed the placenta, and continue to expand and mature in the fetal liver. Before birth, cycling HSCs migrate from the liver to the developing BM. At the end of the preadult stage, organ growth slows down and eventually stops. Stem cell quiescence coincides with the switch from developmental expansion to homeostatic maintenance. $P$-Sp para-aortic splanchnopleural mesoderm; AGM aortagonad-mesonephros. (b) HSCs actively cycle and expand during development. (c) HSCs are kept quiescent in the adult BM niche. HSCs divide symmetrically or asymmetrically. Differentiated daughter cells continue to differentiate into mature cells, whereas the copy of the parent cell is kept in the niche. In the presence of stress, the niche environment may be changed to activate HSC division 


\subsection{The Hematopoietic Stem Cell Niche}

\subsubsection{Niche Cell Components}

\subsubsection{Two Types of Niches}

The pathways via which two different types of HSCs, quiescent and cycling, are maintained in the HSC niche in the BM are of great interest. A dynamic model of hematopoiesis has been proposed, (Arai and Suda 2007; Iwasaki and Suda 2009) wherein quiescent HSCs are located and maintained on the surface of the endosteum during homeostasis. Occasionally, these cells move to the perivascular niche when needed and become activated by extrinsic signals to enter the cell cycle. Subsequently, cycling HSCs divide and provide peripheral blood with mature cells. When the supply of mature cells is no longer needed, activated HSCs located in the perivascular niche return to the osteoblastic niche on the surface of endosteum, where they fall back to the quiescent state and are maintained (Fig. 2.2a).

Osteoblasts are bone-forming cells that are derived from mesenchymal stem cells (MSCs), which are the main population of cells covering the endosteal surface. Osteoblasts are prime candidates for HSC niche cells based on several studies:
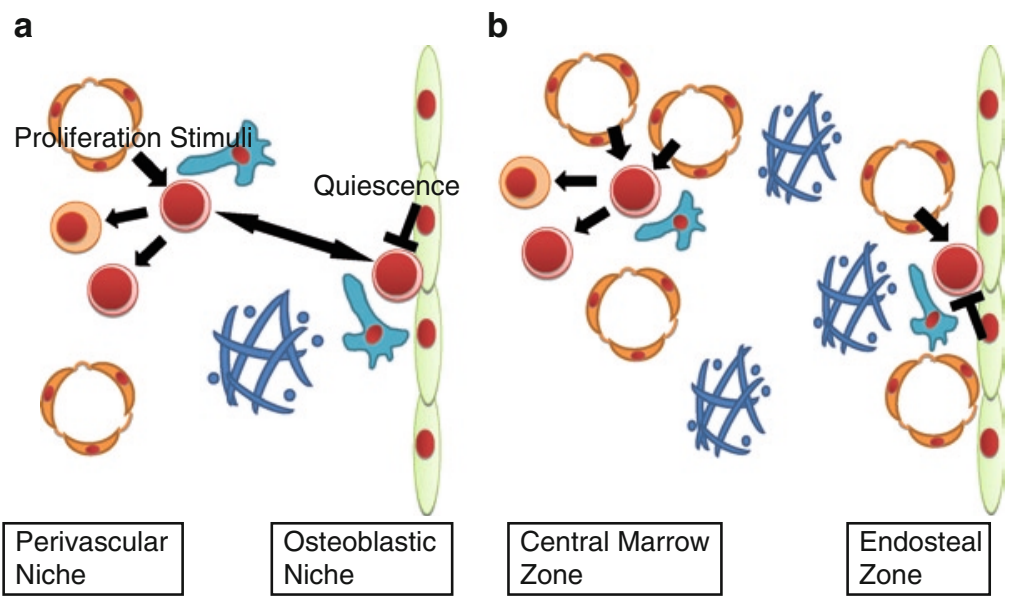

Fig. 2.2 Niche structure models. (a) The "two types of niche model" involves the osteoblastic niche and the perivascular niche. Quiescent HSCs are maintained in the osteoblastic niche. When needed HSCs exit from the endosteal area and migrate into the perivascular niche where their cell cycle is activated. As a result, HSC proliferation and differentiation occur to produce mature cells. HSCs shuttle between the two types of niche. (b) Recent investigations revealed the presence of close interactions between the osteoblastic and endothelial niches. Microvessels exist near the endosteum. HSCs receive inhibitory signals that block the cell cycle from the cells located on the endosteal surface and activation signals from the endothelial cells located at the endosteal zone. In contrast, HSCs are exposed mainly to the activation signals from the endothelial cells located at the central marrow zone 
osteoblasts secrete cytokines that modulate stem cell function, stimulate the growth of hematopoietic cells in in vitro culture, and promote BM engraftment of HSCs in vivo when cotransplanted (El-Badri et al. 1998; Taichman 2005). The groups of $\mathrm{Li}$ and Scadden demonstrated that osteoblasts are a HSC niche in in vivo studies (Calvi et al. 2003; Zhang et al. 2003). They showed that the number of HSCs is controlled by the number of osteoblasts, i.e., niche size. The Li group demonstrated that the BMP signaling through the BMP receptor type IA in osteoblasts controls the number of osteoblasts that express N-cadherin, which correlates with the number of HSCs. These authors also showed via histological analysis that a subset of osteoblasts expressing high levels of $\mathrm{N}$-cadherin function as a key component of the osteoblastic niche. Scadden and his colleague stimulated osteoblasts using activated parathyroid hormone/parathyroid hormone-related peptide (PTH/PTHrP) receptors (PPRs). The stimulated osteoblasts increased in number and produced high levels of the Notch ligand jagged 1, which led to an increase in the number of HSCs. Visnjic et al. confirmed these results in a transgenic mouse model wherein expression of the herpes virus thymidine kinase gene is under the control of the collagen $\alpha$ 1-type promoter. In this model, hematopoiesis was severely suppressed following conditional ablation of osteoblasts by exposing mice to ganciclovir (Visnjic et al. 2004).

The osteoblastic niche on the endosteal surface comprises not only mature osteoblasts, but also multiple subsets of endosteal cell populations, which may act synergistically to form a complex that supports the HSCs located at the endosteum. Osteoblasts are a heterogeneous population that includes immature and mature cells (Kiel and Morrison 2008). We performed a precise investigation of the osteoblasts that cover the BM endosteum, and identified an osteoblastic population that most effectively enhances the long-term repopulation activity of HSCs. Gene expression profiling also revealed the heterogeneity of endosteal cell populations (Nakamura et al. 2010).

The perivascular niche is composed mainly of endothelial cells of the sinusoid. Hematopoietic and endothelial cells are derived from a common progenitor, the hemangioblast (Choi et al. 1998). Both HSCs and endothelial cells express CD31, CD34, CD133 (Prominin-A), fetal liver kinase1 (Flk1), and endothelium-specific receptor tyrosine kinase 2 (Tie2) (Takakura et al. 1998; Rafii et al. 2002). During embryonic development, HSCs and endothelial cells are tightly linked, and studies of this stage have taken the lead in the area of perivascular niche research. It has been shown that primary endothelial cells in the adult liver are able to support hematopoietic progenitors in culture (Cardier and Barbera-Guillem 1997). Heissig et al. proposed that after BM ablation, hematopoietic progenitors are translocated to the perivascular niche, which is initiated by soluble Kit ligand cleaved by matrix metalloproteinase-9 (MMP-9) (Heissig et al. 2002).

The Morrison group conducted a study using antibodies against the signaling lymphocyte activation molecule (SLAM) family of receptors, to distinguish HSCs from progenitor cells in the BM, and found that HSCs are highly concentrated in the $\mathrm{CD} 150^{+} \mathrm{CD} 244^{-} \mathrm{CD} 48^{-}$population (Kiel et al. 2005). The ability to purify HSCs based on a simple combination of SLAM receptors allowed the relatively easy identification of HSCs in tissue sections. In their histological study, the majority of 
CD150+CD244-CD48- cells were found near the sinusoidal endothelium in the spleen and BM, although some were associated with the endosteum. Cells located near sinusoids are exposed to many factors streaming in the peripheral blood, such as hormones, growth factors, oxygen, and nutrients. Therefore, HSCs located in the perivascular niche can sensitively perceive signal changes according to homeostatic need and be activated for proliferation and differentiation to produce mature blood cells.

Cells other than osteoblasts and endothelial cells exist in the BM. Recent work suggests that the other populations also participate in the regulation of HSCs. Sugiyama et al. found that most HSCs were in contact with reticular cells expressing a high amount of chemokine (C-X-C motif) ligand 12 (CXCL12, also known as stromal cell-derived factor-1 [SDF-1]), the ligand for chemokine (C-X-C motif) receptor 4 (CXCR4) (Sugiyama et al. 2006). These cells are called CXCL12abundant reticular (CAR) cells. Severe reduction in number of HSC and higher sensitivity to myelotoxic injury were observed in the absence of CXCL12-CXCR4 signaling. CAR cells are present both in vascular and endosteal niches, although the origin of CAR cells remains unknown.

Katayama et al. demonstrated that the sympathetic nervous system, which secretes norepinephrine (NE), regulates the attraction of stem cells to their niche by controlling the BM niche (Katayama et al. 2006). In UDP-galactose ceramide galactosyltransferase-deficient $\left(\mathrm{Cgt}^{-/-}\right)$mice, which exhibit abnormal nerve conduction, no hematopoietic stem and progenitor cells egress from the BM after induction of migration. Further studies indicated that NE mediates the suppression of osteoblasts and the downregulation of bone CXCL12 that are induced by granulocyte colony-stimulating factor (G-CSF). These modifications result in changes in hematopoietic stem and progenitor cell mobilization.

Osteoclasts and adipocytes may also take part in the regulation of HSCs, although the function of these cells in the BM niche is not well understood. Strontium ( $\mathrm{Sr}$ ) is a bone anabolic agent that increases bone and osteoblast formation and inhibits osteoclast activity. BMT into Sr-treated mice led to a delay in hematopoietic recovery despite the increase in osteoblastic activity (Lymperi et al. 2008). In another study, Kollet et al. stimulated osteoclasts using several stresses and demonstrated that activation of osteoclasts increases the recruitment of hematopoietic progenitors into the circulation (Kollet et al. 2006). Adipocytes are enriched in the BM, especially in the elderly. Naveiras et al. focused on the difference in adipocyte content between the vertebrae of the tail and the thorax and demonstrated that adipocytes were enriched in vertebrae of the tail where HSCs and short-term progenitors were reduced (Naveiras et al. 2009). In A-ZIP/F1 mice, which are fatless, marrow engraftment was accelerated compared with wild-type animals. These data indicate that adipocytes may act as negative regulators of the HSC niche while adiponectin, which is secreted mainly from adipocyte, reportedly functions as a positive regulator of HSCs (DiMascio et al. 2007).

The identification of MSCs, the progeny of which constitutes the BM niche, has led to further progress in investigation of the HSC niche compartment. The concurrent formation of the hematopoietic environment and of heterotopic bone via 
in vivo transplantation of BM fragments or BM stromal cells into an ectopic site suggests that HSCs and MSCs coexist in the BM. Sacchetti et al. showed that melanoma-associated cell adhesion molecule (MCAM) (also known as CD146)expressing subendothelial cells in human BM can organize an ectopic hematopoietic microenvironment within a miniature bone organ (Sacchetti et al. 2007). These subendothelial cells maintain the self-renewal capacity in the ectopic hematopoietic region. This result suggests the subendothelial cells that express MCAM may be MSCs. Another group led by Weissman sorted cells collected from fetal bones (at 15.5 days postcoitum) and identified a population of progenitor cells that expressed the surface markers CD45- Tie2 ${ }^{-} \alpha_{\mathrm{V}}{ }^{+} \mathrm{CD} 105^{+}$Thy $1.1^{-}$(CD105+Thy1.1-) and produced donor-derived ectopic bones when transplanted under the adult mouse kidney capsule (Chan et al. 2009). The ectopic bone was generated through a cartilage intermediate, and a marrow cavity populated by host-derived long-term HSCs was also generated within this structure. Transplantation of CD105'Thy1.1- cells with concomitant suppression of the expression of factors involved in endochondral ossification in the host mice resulted in the absence of niche formation. This result indicates the necessity of endochondral ossification for the formation of the BM niche.

\subsubsection{Close Interaction Between the Endosteal and Perivascular Zones}

As described above, there are many types of HSC-supporting cells in the BM. Therefore, it is difficult to define two distinct BM niches according to the types of the cells involved in the formation of niche. Close interaction between the osteoblastic and perivascular niches has been identified using new imaging technologies. The Scadden group observed HSCs at the single-cell level using a combinational method of high-resolution confocal microscopy and two-photon video imaging, and found that osteoblasts were enmeshed in microvessels and formed a complex tissue (Lo Celso et al. 2009). After transplantation into irradiated or c-Kit receptor deficient mice, HSCs and progenitors localized near endosteum, while more differentiated cells stayed at distant locations from endosteum. Under physiological challenge of BMT, which demands engraftment or expansion of HSCs, HSCs and progenitors moved into positions near endosteum and osteoblasts. The Li group traced the migration of $\mathrm{GFP}^{+}$HSCs using a newly developed real-time ex vivo imaging technology (Xie et al. 2009). They demonstrated that HSCs tended to migrate to the endosteum when transplanted to irradiated mice and a fraction of these cells divided actively. In contrast, HSCs were located randomly in BM when transplanted in nonirradiated mice. In this study, the authors observed the presence of well-vascularized endosteum and vasculatures near $\mathrm{N}$-cadherin ${ }^{+}$preosteoblasts. These results indicate that HSCs located in the endosteal zone interact with both endosteal and endothelial cells and are prompted to expand in the endosteum zone in response to BM damage, which means that the osteoblastic and perivascular niches cannot be distinguished clearly. Therefore, Li and his colleagues proposed an alternate concept for two types of BM niches, i.e., the endosteal zone and the 
central marrow zone. They suggest that, although the endosteal zone normally sends inhibitory signals for cell cycling to maintain HSC quiescence, it transiently turns into a stimulatory environment when the BM is damaged (Fig. 2.2b).

\subsubsection{Niche Factors}

Given the complexity of the niche structure and its cellular components, it is not only important to analyze niche functions based on its physical structure but also by the factors that are produced by the niche components themselves. Each type of niche cell exhibits a specific gene expression pattern, which may lead to secretion of a specific combination of potential niche factors for HSC maintenance. The BM also is comprised of extracellular matrix (ECM) and each ECM component may also contribute to the niche. Therefore, to fully understand the characteristics of the niche, research should focus on adhesion molecules, cytokines, growth factors, and some local environmental factors (Fig. 2.3).

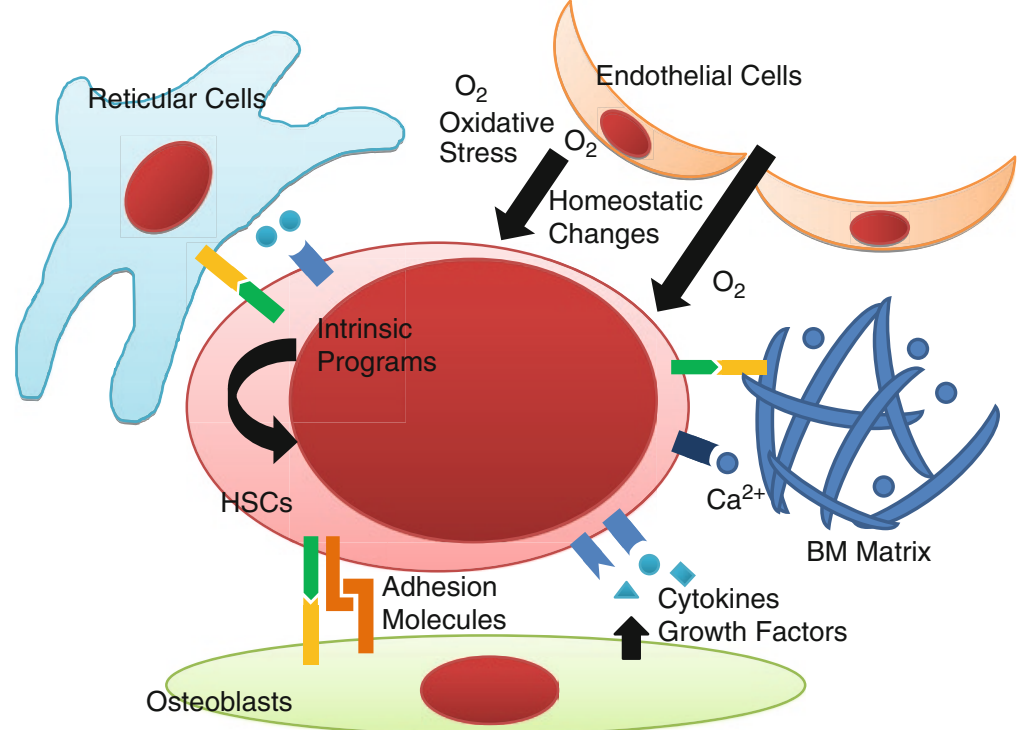

Fig. 2.3 Components of the hematopoietic niche in BM. HSCs are maintained by intrinsic and extrinsic stimuli. HSCs attach to niche cells and to the BM matrix via adhesion molecules, some of which also activate signals for HSC maintenance. BM cells secrete cytokines and growth factors, such as Wnt, MPL, and CXCL12. Oxygen concentration is higher around the sinusoid. Signals from peripheral blood reflect homeostatic changes. $\mathrm{Ca}^{2+}$ is abundant in the $\mathrm{BM}$ and regulates HSCs. Intrinsic programs, especially those associated with transcription factors such as Hox and Evi-1, also regulate HSC fate 


\subsubsection{Adhesion Molecules}

One of the primary functions of the niche is to physically interact with stem cells. HSCs and their niche cells attach to each other through adhesion molecules, which are also important for lodging and engraftment. Niche cells express various adhesion molecules including $\mathrm{N}$-cadherin, which is a hemophilic adhesion molecules and binds to $\mathrm{N}$-cadherin expressed on HSCs, intercellular adhesion molecule 1 (ICAM-1), which binds to integrin alpha-L (LFA-1A) expressed by HSCs, vascular cell adhesion molecule 1 (VCAM-1) that binds to integrin alpha-4 (VLA-4) expressed by osteoblasts, and VCAM-1 that binds with VLA-4 on endothelial cells (Wilson and Trumpp 2006).

The Li group, which had identified the osteoblastic niche (composed of $\mathrm{N}$-cadherin ${ }^{+}$osteoblasts), observed the presence of $\mathrm{N}$-cadherin/ $\beta$ catenin complexes between HSCs and osteoblasts (Zhang et al. 2003). This result suggests that $\mathrm{N}_{\text {-cadherin }}{ }^{+}$osteoblasts support HSCs via this adhesive interaction. N-cadherin is a representative adhesion molecule in the osteoblastic niche and is the only member of the cadherin family that is expressed in both quiescent HSCs and osteoblasts (Arai et al. 2004).

Integrins are also important adhesion molecules in the HSC niche. In particular, integrin-superfamily heterodimers, VLA-4 and integrin $\alpha 5 \beta 1$ (VLA-5), are expressed in HSCs, which bind to fibronectin and osteopontin (Opn) in the BM ECM, and to VCAM-1 on the niche cell surface (Whetton and Graham 1999; Wilson and Trumpp 2006). Binding to fibronectin regulates the expression of specific transcription factors that have roles in stem cell maintenance (Dao and Nolta 2007). For example, human CD34+ cells cultured on a fibronectin peptide containing the binding site for VLA-4 and VLA-5 expressed higher levels of the protooncogene protein c-Myb and endothelial transcription factor GATA-2 in vitro, which are known to be abundant in primitive cells, as compared to cells grown in suspension culture. The cells also expressed lower levels of p21 and the transcription factor PU.1, which are upregulated during monocyte and myeloid differentiation, respectively. The interaction between VLA-4 and VCAM-1 is also important for lodging and engraftment at the endothelial niche (Papayannopoulou et al. 1995). We demonstrated that the interaction between Tie2, which is expressed in HSCs, and angiopoietin-1 (Ang1), which is produced by the osteoblasts, activates $\beta 1$ integrin and $\mathrm{N}$-cadherin and that this enhanced adhesion mediates the maintenance of stem cell quiescence in the osteoblastic niche (Arai et al. 2004).

The osteoblastic niche sends inhibitory signals to HSCs to prevent their proliferation and maintain their quiescence. In some cases, adhesion molecules themselves send inhibitory signals to HSCs. For example, $\mathrm{N}$-cadherin inhibits the activity of $\beta$-catenin, which is the key intercellular molecule in the Wnt signaling pathway (Wilson and Trumpp 2006).

Opn, a matrix glycoprotein that is exclusively produced by osteoblasts on the endosteal bone surface, is a key adhesion component of the osteoblastic niche (Nilsson et al. 2005; Stier et al. 2005; Adams and Scadden 2006). Opn binds to VLA-4, VLA-5, and CD44 expressed in HSCs, which supports the adhesion of 
HSCs to the osteoblastic niche. In addition, Opn acts as a negative regulator of HSC proliferation. Markedly enhanced cycling of HSCs was observed in $\mathrm{Opn}^{-1-}$ mice and the proliferation of hematopoietic stem/progenitor cells was suppressed by exogenous Opn in vitro (Nilsson et al. 2005). The Scadden group stimulated the $\mathrm{BM}$ niche in $\mathrm{Opn}^{-/-}$mice using parathyroid hormone and observed an abnormal increase in the number of HSCs (Stier et al. 2005; Adams and Scadden 2006). Therefore, when the osteoblastic niche is activated Opn may function to restrict excessive stem cell expansion.

Finally, CD44 is a cell surface receptor expressed on HSCs that binds to the glycosaminoglycan hyaluronic acid (HA), which is a major component of the BM ECM. The CD44/HA interaction has also been shown to play important roles in modulating the migration and anchorage of HSCs in the BM niche (Avigdor et al. 2004; Adams and Scadden 2006).

\subsubsection{Cytokines and Growth Factors}

Molecular signaling pathways that regulate stem cell function in the niche of the GSCs of Drosophila may be simpler compared with those observed in mammal niches. In the fly ovariole, GSCs are mainly and strictly governed by BMP signaling (Chen and McKearin 2003; Song et al. 2004). In contrast, in Drosophila testis hub cells support the self-renew and maintenance of GSCs by expressing unpaired (UPD), which activates the Janus kinase (JAK)/signal transducer and activator of transcription (STAT) pathway (Kiger et al. 2001; Tulina and Matunis 2001).

In contrast with what is observed in the GSCs of Drosophila, HSCs in mammals are maintained by multiple regulators expressed in the niche. Extrinsic signaling pathways, such as the Wnt and Notch pathways play fundamental roles in the niche-mediated regulation of HSCs (Reya et al. 2003; Duncan et al. 2005). These signals are also important for development. Overreaching signals, however, are detrimental. For example, although Wnt signaling was first reported to expand HSCs while maintaining their function (Reya et al. 2003) further investigation revealed that an overabundance of Wnt signaling leads to exhaustion of the longterm HSC pool (Kirstetter et al. 2006; Scheller et al. 2006). In contrast, an in vivo study demonstrated that the simultaneous deficiency of $\beta$-catenin, an essential factor for Wnt signaling, and $\gamma$-catenin, a close homolog of $\beta$-catenin, in conditional knockout (KO) mice did not impair HSC function (Koch et al. 2008). Similarly, adult HSCs can be maintained even under inactivation of Notch1 and its receptor Jagged1 (Mancini et al. 2005). In mammals, maintenance of stem cells and, ultimately, the organs in which they reside may not totally depend upon any single signal and different signals may compensate for each other in the case of functional impairment. Additionally, some signaling pathways impinge specifically on niche cells. For example, Zhang et al. showed that BMP signaling in osteoblasts controls the number of osteoblasts themselves, which leads to the regulation of the number of HSCs (Zhang et al. 2003).

Several cytokines and growth factors that are expressed by niche cells act on HSCs. Thrombopoietin (THPO), which is the ligand of the myeloproliferative 
leukemia virus oncogene protein $(\mathrm{Mpl})$, is a representative factor in the HSC niche. It also regulates megakaryogenesis. Jacobsen and our groups demonstrated that THPO/Mpl signaling is an important pathway in the regulation of HSC maintenance in the osteoblastic niche (Qian et al. 2007; Yoshihara et al. 2007). We showed that long-term HSCs that express $\mathrm{Mpl}$ are in close contact with THPO-producing osteoblastic cells, which lead to the maintenance of HSC quiescence. $\beta 1$-integrin and the CDK inhibitor $p 57^{\text {Kip2 } 2}$ are also upregulated in HSCs by THPO/Mpl signaling. The Jacobsen group found a 150-fold decrease in the number of HSCs in adult $\mathrm{Thpo}^{-/}$mice. Moreover, HSCs in these mice were induced to enter the cell cycle and mRNA expression levels of the CDK inhibitors $p 57^{\mathrm{Kip} 2}, p 19^{\text {INK4D }}$ as well as multiple Hox transcription factors were downregulated. They also demonstrated that fetal HSC expansion does not require THPO/Mpl signaling.

CXCL12/CXCR4 signaling plays a critical role in hematopoiesis during ontology (Ara et al. 2003). Cxcl12 $2^{-/-}$mouse embryos exhibit a severe impairment in the homing of the HSCs into BM after their expansion in fetal liver and of the myeloid cells. As described by Sugiyama et al., CXCL12/CXCR4 signaling plays an essential role in the maintenance of HSCs in the adult BM (Sugiyama et al. 2006). CXCL12/ CXCR4 signaling also regulates trafficking and mobilization of HSCs. Furthermore, this signaling has a role not only in hematopoiesis but also in metastasis and engraftment mechanism of malignant cells in BM (Zhang and Li 2008). Thus, CXCL12/CXCR4 signaling has multiple functions and is active in many types of cells, and as such deserves a greater level of attention and investigation.

\subsubsection{Local Environmental Factors}

Local environmental factors are also important niche factors. One of the major local components of the niche is oxygen, as the concentration of this molecule directly affects the production level of reactive oxygen species (ROS) in HSCs. The superoxide anion radical, hydrogen peroxide, and the hydroxyl radical are important ROS. These molecules can act as free radicals and cause DNA damage (Naka et al. 2008). Quiescent HSCs are kept in a hypoxic environment and protected from ROS-mediated oxidative damage. Increased ROS levels in multipotent HSCs in Drosophila cause precocious differentiation into mature red blood cells (OwusuAnsah and Banerjee 2009).

Jang and Sharkis performed serial transplantations to demonstrate that HSCs with lower intercellular ROS level have a higher self-renewal potential (Jang and Sharkis 2007). In contrast, HSCs of the ROS ${ }^{\text {high }}$ population were exhausted during serial transplantation, which was rescued by treatment with the antioxidation agent $\mathrm{N}$-acetyl-L-cysteine (NAC). This suggests that intracellular ROS levels play a crucial role in the control of the long-term self-renewal ability of HSCs.

The ataxia telangiectasia mutated (Atm) gene plays a role in genomic stability. Atm activates a key cell cycle checkpoint in response to DNA damage, telomeric instability, and oxidative stress. In $\mathrm{Atm}^{-/}$mice, a smaller proportion of cells are found in the stem cell fractions. Colony formation capacity after long-term culture and long-term BM reconstitution capacities were also impaired in hematopoietic cells from $\mathrm{Atm}^{-/-}$mice 
(Ito et al. 2004). Progressive BM failure following the defective maintenance of the adult HSC pool was observed in $\mathrm{Atm}^{-/-}$mice older than 24 weeks. This was associated with elevated ROS levels, and treatment with NAC recovered the function of $\mathrm{Atm}^{-/-}$HSCs and rescued the mice from BM failure. Further investigation revealed that the defect in the maintenance of HSC quiescence in $\mathrm{Atm}^{-/-}$mice resulted from phosphorylation of the p38 mitogen-activated protein kinase (MAPK) in HSCs, which was induced by elevation of ROS levels (Ito et al. 2006).

FoxOs regulate a variety of processes in cells, such as induction of cell cycle arrest, stress resistance, and apoptosis. To assess the role of FoxO family members in hematopoiesis, Tothova et al. deleted three FoxOs (FoxO1, FoxO3, and FoxO4) in mice in a conditional manner (Tothova et al. 2007). The deletion of these FoxOs led to the expansion of myeloid lineages and to the decrease in the number of hematopoietic stem/progenitor cells. Defective long-term repopulation activity with activated cell cycling, apoptosis, increased accumulation of ROS, and changes in the expression of genes that regulate ROS were also observed in HSCs in FoxO-null mice. These phenotypes were rescued by treatment with NAC, which suggests that FoxO proteins prevent the accumulation of oxidative stress (or ROS) in HSCs and thereby mediate the quiescence and maintenance of HSCs. Among all, FoxO3 is the most highly expressed FoxO in the hematopoietic cells in BM. Detailed analysis of $\mathrm{FoxO}^{-/-}$mice revealed that HSCs from these mice had a decreased ability to support long-term reconstitution of hematopoiesis (Miyamoto et al. 2007). FoxO3 ${ }^{-/-}$HSCs did not remain in quiescence, were highly sensitive to cell cycle-dependent myelotoxic injury, and exhibited elevation of ROS levels. Furthermore, the defects in the activities of FoxO3 $^{-1-}$ HSCs were rescued by NAC treatment (Yalcin et al. 2008). These studies collectively suggest that $\mathrm{FoxO} 3$ is a key factor for maintaining HSC quiescence. The endosteal surface may be more hypoxic than the perivascular zone, and therefore, the endosteal zone seems to be better suited for maintaining quiescence of HSCs.

Signals received from the simple mineral content of the niche also regulate the behavior of HSCs. Calcium-sensing receptor ( Casr) expressed on HSCs recognizes concentrations of calcium ions on the endosteal surface. In $\mathrm{Casr}^{-/-}$mice, HSCs in fetal liver show normal characteristics, and migrate and home to the BM (Adams et al. 2006). However, $\mathrm{Casr}^{-/}$antenatal mice have few primitive hematopoietic cells in the BM because of defects in the ability of HSC to localize to the osteoblastic niche and to adhere to the ECM protein collagen I.

\subsection{Intrinsic Programs in HSCs}

The autonomous maintenance activity is essential for sustaining healthy HSCs. HSCs exhibit some characteristic expression profile of genes; for example, transcription factors which are expressed at a high level uniquely in HSCs play 
important roles in determining their biological characteristics. These transcription factors may be divided into two categories; those that are required for selfrenewal and maintenance of $\mathrm{HSCs}$ and those necessary for lineage-specific differentiation. Although the strict grouping of transcription factors into these two categories is difficult, runt related transcription factor 1 (Runxl) (Growney et al. 2005), ets variant gene 6 (Etv6, also known as Tel) (Hock et al. 2004b), T-cell acute lymphocytic leukemia 1 (Tall, also known as Scl) (Endoh et al. 2002), homeobox B4 (Hoxb4) (Kyba et al. 2002), and Ecotropic viral integration site-1 (Evil) (Goyama et al. 2008) have been shown to be critical for HSC selfrenewal, maintenance, or both.

Homeobox transcription factors are important regulators of primitive hematopoietic cell proliferation and differentiation. In particular, Hoxb4 is a strong positive regulator of HSC self-renewal. Antonchuk et al. demonstrated that over expression of Hoxb4 in HSCs induces cell expansion in vitro while retaining the repopulating capacity (Antonchuk et al. 2002).

Evil is a member of the SET/PR domain family of zinc-finger transcription factors. The number of HSCs was severely reduced in $\mathrm{Evil}^{-/-}$mouse embryos, which was accompanied by defects in the proliferative and repopulating capacities of these cells (Goyama et al. 2008). As a result, hematopoiesis was not maintained in Evi1 ${ }^{-1-}$ adult mice.

Bmil polycomb ring finger oncogene (Bmil) also plays an important role in transcription. Its product, the polycomb complex protein Bmi-1, is essential for the self-renewal of hematopoietic and neural stem cells and its autonomous activity has been extensively studied. $\mathrm{Bmil}^{-/-}$postnatal mice exhibited a remarkable reduction in the number of HSCs (Park et al. 2003). Moreover, there was a complete lack of HSC self-renewal capacity in $\mathrm{Bmil}^{-/-}$adult mice. In addition, the expression pattern of genes associated with stem cell survival, transcription, or proliferation was altered in $\mathrm{Bmil}^{-/-} \mathrm{BM}$ mononuclear cells.

Expressions of a certain transcription factors is not only differentiation stagespecific but often also developmental stage-specific. For example, the transcription factor Soxl7 is expressed in HSCs of fetal liver and in neonatal BM but downregulated in adult BM (Kim et al. 2007). In contrast, other transcription factors, such as Gfi-1 (Hock et al. 2004a) and Tel/Etv6 (Hock et al. 2004b), are important for the self-renewal and maintenance of adult HSCs but not during fetal development. It may be important to segregate the supportive molecular mechanisms that influence the capacities of HSCs, according to the physiological needs in each developmental stage.

In some tissue stem cell systems, the intrinsic program is sufficient for stem cell maintenance and differentiation. The anatomy of the intestinal crypt is uniquely suited for the study of tissue stem cells in their niche. Crypt base columnar cells which express Lgr5 are not quiescent stem cells; rather, they are stem cells dividing everyday (Barker et al. 2007). The Clevers group showed that single $\mathrm{Lgr}^{+}$build crypt - villus structures in vitro in the absence of the niche (Sato et al. 2009a). 


\subsection{Mobilization of Normal and Leukemic Stem Cells}

Stem cells are mobilized from the niche. This process is not purely stochastic. Rather, it is well regulated by extrinsic signals that are derived from the surrounding cells. The decrease in the number of blood cells following the administration of cytotoxic drugs, especially the decrease of progenitor cells, initiate the exit of quiescent HSCs from the niche and their proliferation to fulfill the necessary mature cells.

Treatment of mice with G-CSF induces the efficient mobilization of HSCs to the peripheral blood. This mobilization includes serial events: exit of HSCs from the niche, egression from the BM, and shift to the marginal pool in the blood vessel. G-CSF has been shown to induce the release of proteolytic enzymes that cleave adhesion molecules, such as VCAM1, VLA-4, and CXCL12 (Petit et al. 2002). ROS may also be involved in the release of stem cells from the niche via the downregulation of adhesion molecules in HSCs (Hosokawa et al. 2007). Thus, stem cell mobilization is triggered by altering the niche microenvironment. Therefore, understanding the regulation of the niche will be important to improve BMT methodology or for developing cancer treatments that target quiescent cancer stem cells (CSCs).

Cancer tissues comprise stem cell-like cells (Bonnet and Dick 1997), which mimic the phenotypes and behavior of normal stem cells. Dick et al. termed these cells as CSCs or cancer-initiating cells (see Chap. 10). Small numbers of human CSCs can give rise to tumors in immunodeficient mice, whereas other progenitorlike cancer cells do not. CSCs exhibit niche dependency and slow cell cycling, similar to the normal stem cells (Ishikawa et al. 2007). These CSCs are chemoresistant and considered as the cause of relapse and metastasis.

Chronic myeloid leukemia (CML) is a chronic myeloproliferative disorder caused by the transformation of HSCs. Ito et al. investigated whether the treatment outcome of CML could be improved by forcing the quiescent leukemic stem cells (LSCs) into a cycling state using arsenic trioxide, which is a strong ROS generator (Ito et al. 2008). The number of quiescent LSCs decreased significantly after the arsenic treatment. More LSCs entered the cell cycle compared with normal stem cells after treatment with arsenic trioxide, which resulted in good effectiveness of chemotherapy in the mouse model. Similarly, interferon $\alpha$ (IFN $\alpha$ ) signaling induces HSCs to enter the cell cycle (Essers et al. 2009; Sato et al. 2009b). Experiments performed in mice suggest that 5-FU-resistant normal HSCs can be killed efficiently by a 1-3 day pretreatment with IFN $\alpha$. These data suggest that IFN $\alpha$ may also activate and sensitize LSCs for subsequent elimination by tyrosine kinase inhibitors, such as Imatinib. Exiting the niche leads to active cell cycling, and therefore, the combination of chemotherapy or radiation with the artificial induction of HSC mobilization may improve the therapeutic outcome in treatment of leukemia. However, the first pilot studies using G-CSF for LSC cycling did not show the effectiveness of this strategy (Drummond et al. 2009), and further optimization and improvement in protocols may be necessary (Fig. 2.4). 
a

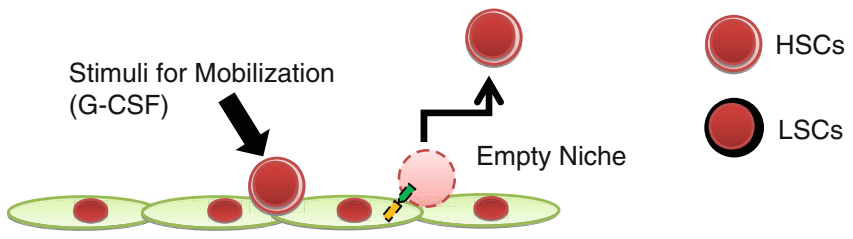

b

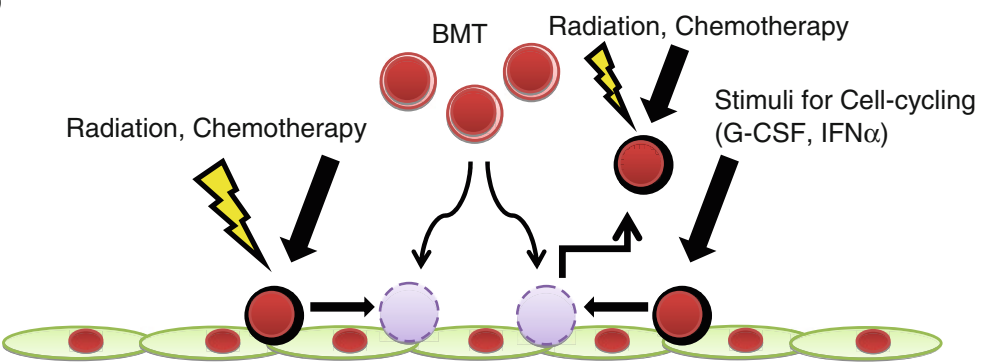

Fig. 2.4 A schema of HSC mobilization. (a) Mobilization stimuli (e.g., G-CSF) lead to the migration of HSCs from their BM niche, which results in empty niches. This characteristic can be applied to the therapy of leukemia. (b) LSCs mimic HSCs and reside in the BM niche to maintain the quiescent state. Radiation therapy or chemotherapy alone will not completely purge the cancer cells as some quiescent LSCs may survive and remain quiescent in the BM niche. These surviving LSCs may cause relapse of the disease. It may be possible to target these LSCs more effectively if they come out of niche and their cell cycle is activated. Development of efficient and safe methods to manipulate LSC maintenance by the niche is desired

\subsection{Concluding Remarks}

The niche is a cradle for stem cells and supplies both a special microenvironment and soluble factors that regulate stem cell function. In addition, under conditions that limit the supply of mature cells, niche cells and the factors they secrete can be modulated to activate stem cells and accelerate their differentiation.

The importance of niche regulation has been demonstrated in a disease model. Retinoic acid receptor $\gamma(R A R \gamma)$-deficient niche may lead to myeloproliferative syndromes, without any intrinsic defects in hematopoietic cells (Walkley et al. 2007). It has been reported that regulations by microenvironments are also involved in the development, maintenance, proliferation, and even metastasis of CSCs (Iwasaki and Suda 2009). These ongoing investigations suggest that the niche could be an important target for cancer therapies. From a biological and clinical perspective, the study of stem cell niches and their intrinsic programs is a promising field for the understanding of the nature of adult stem cells. 


\section{References}

Adams GB, Scadden DT (2006) The hematopoietic stem cell in its place. Nat Immunol 7:333-337.

Adams GB, Chabner KT, Alley IR et al (2006) Stem cell engraftment at the endosteal niche is specified by the calcium-sensing receptor. Nature 439:599-603.

Antonchuk J, Sauvageau G, Humphries RK (2002) HOXB4-induced expansion of adult hematopoietic stem cells ex vivo. Cell 109:39-45.

Ara T, Tokoyoda K, Sugiyama T et al (2003) Long-term hematopoietic stem cells require stromal cell-derived factor-1 for colonizing bone marrow during ontogeny. Immunity 19:257-267.

Arai F, Suda T (2007) Maintenance of quiescent hematopoietic stem cells in the osteoblastic niche. Ann N Y Acad Sci 1106:41-53.

Arai F, Hirao A, Ohmura M et al (2004) Tie2/angiopoietin-1 signaling regulates hematopoietic stem cell quiescence in the bone marrow niche. Cell 118:149-161.

Avigdor A, Goichberg P, Shivtiel S et al (2004) CD44 and hyaluronic acid cooperate with SDF-1 in the trafficking of human CD34+ stem/progenitor cells to bone marrow. Blood 103:2981-2989.

Barker N, van Es JH, Kuipers J et al (2007) Identification of stem cells in small intestine and colon by marker gene Lgr5. Nature 449:1003-1007.

Barker N, Huch M, Kujala P et al (2010) Lgr5(+ve) stem cells drive self-renewal in the stomach and build long-lived gastric units in vitro. Cell Stem Cell 6:25-36.

Bonnet D, Dick JE (1997) Human acute myeloid leukemia is organized as a hierarchy that originates from a primitive hematopoietic cell. Nat Med 3:730-737.

Bradford GB, Williams B, Rossi R et al (1997) Quiescence, cycling, and turnover in the primitive hematopoietic stem cell compartment. Exp Hematol 25:445-453.

Calvi LM, Adams GB, Weibrecht KW et al (2003) Osteoblastic cells regulate the haematopoietic stem cell niche. Nature 425:841-846.

Cardier JE, Barbera-Guillem E (1997) Extramedullary hematopoiesis in the adult mouse liver is associated with specific hepatic sinusoidal endothelial cells. Hepatology 26:165-175.

Chan CK, Chen CC, Luppen CA et al (2009) Endochondral ossification is required for haematopoietic stem-cell niche formation. Nature 457:490-494.

Chen D, McKearin D (2003) Dpp signaling silences bam transcription directly to establish asymmetric divisions of germline stem cells. Curr Biol 13:1786-1791.

Cheng T, Rodrigues N, Shen H et al (2000) Hematopoietic stem cell quiescence maintained by p21cip1/waf1. Science 287:1804-1808.

Choi K, Kennedy M, Kazarov A et al (1998) A common precursor for hematopoietic and endothelial cells. Development 125:725-732.

Dao MA, Nolta JA (2007) Cytokine and integrin stimulation synergize to promote higher levels of GATA-2, c-myb, and CD34 protein in primary human hematopoietic progenitors from bone marrow. Blood 109:2373-2379.

Deng W, Lin H (1997) Spectrosomes and fusomes anchor mitotic spindles during asymmetric germ cell divisions and facilitate the formation of a polarized microtubule array for oocyte specification in Drosophila. Dev Biol 189:79-94.

DiMascio L, Voermans C, Uqoezwa M et al (2007) Identification of adiponectin as a novel hemopoietic stem cell growth factor. J Immunol 178:3511-3520.

Drummond MW, Heaney N, Kaeda J et al (2009) A pilot study of continuous imatinib vs pulsed imatinib with or without G-CSF in CML patients who have achieved a complete cytogenetic response. Leukemia 23:1199-1201.

Duncan AW, Rattis FM, DiMascio LN et al (2005) Integration of Notch and Wnt signaling in hematopoietic stem cell maintenance. Nat Immunol 6:314-322.

El-Badri NS, Wang BY, Cherry et al (1998) Osteoblasts promote engraftment of allogeneic hematopoietic stem cells. Exp Hematol 26:110-116. 
Endoh M, Ogawa M, Orkin S et al (2002) SCL/tal-1-dependent process determines a competence to select the definitive hematopoietic lineage prior to endothelial differentiation. EMBO J 21:6700-6708.

Essers MA, Offner S, Blanco-Bose WE et al (2009) IFNalpha activates dormant haematopoietic stem cells in vivo. Nature 458:904-908.

Foudi A, Hochedlinger K, Van Buren D et al (2009) Analysis of histone 2B-GFP retention reveals slowly cycling hematopoietic stem cells. Nat Biotechnol 27:84-90.

Fuchs E, Segre JA (2000) Stem cells: a new lease on life. Cell 100:143-155.

Fuller MT, Spradling AC (2007) Male and female Drosophila germline stem cells: two versions of immortality. Science 316:402-404.

Goyama S, Yamamoto G, Shimabe M et al (2008) Evi-1 is a critical regulator for hematopoietic stem cells and transformed leukemic cells. Cell Stem Cell 3:207-220.

Growney JD, Shigematsu H, Li Z et al (2005) Loss of Runx1 perturbs adult hematopoiesis and is associated with a myeloproliferative phenotype. Blood 106:494-504.

Heissig B, Hattori K, Dias S et al (2002) Recruitment of stem and progenitor cells from the bone marrow niche requires MMP-9 mediated release of kit-ligand. Cell 109:625-637.

Hock H, Hamblen MJ, Rooke HM et al (2004a) Gfi-1 restricts proliferation and preserves functional integrity of haematopoietic stem cells. Nature 431:1002-1007.

Hock H, Meade E, Medeiros S et al (2004b) Tel/Etv6 is an essential and selective regulator of adult hematopoietic stem cell survival. Genes Dev 18:2336-2341.

Hosokawa K, Arai F, Yoshihara $\mathrm{H}$ et al (2007) Function of oxidative stress in the regulation of hematopoietic stem cell-niche interaction. Biochem Biophys Res Commun 363:578-583.

Ishikawa F, Yoshida S, Saito Y et al (2007) Chemotherapy-resistant human AML stem cells home to and engraft within the bone-marrow endosteal region. Nat Biotechnol 25:1315-1321.

Ito K, Hirao A, Arai F et al (2004) Regulation of oxidative stress by ATM is required for selfrenewal of haematopoietic stem cells. Nature 431:997-1002.

Ito K, Hirao A, Arai F et al (2006) Reactive oxygen species act through p38 MAPK to limit the lifespan of hematopoietic stem cells. Nat Med 12:446-451.

Ito K, Bernardi R, Morotti A et al (2008) PML targeting eradicates quiescent leukaemia-initiating cells. Nature 453:1072-1078.

Iwasaki H, Suda T (2009) Cancer stem cells and their niche. Cancer Sci 100:1166-1172.

Jang YY, Sharkis SJ (2007) A low level of reactive oxygen species selects for primitive hematopoietic stem cells that may reside in the low-oxygenic niche. Blood 110:3056-3063.

Katayama Y, Battista M, Kao WM et al (2006) Signals from the sympathetic nervous system regulate hematopoietic stem cell egress from bone marrow. Cell 124:407-421.

Kiel MJ, Morrison SJ (2008) Uncertainty in the niches that maintain haematopoietic stem cells. Nat Rev Immunol 8:290-301.

Kiel MJ, Yilmaz OH, Iwashita T et al (2005) SLAM family receptors distinguish hematopoietic stem and progenitor cells and reveal endothelial niches for stem cells. Cell 121:1109-1121.

Kiger AA, Jones DL, Schulz C et al (2001) Stem cell self-renewal specified by JAK-STAT activation in response to a support cell cue. Science 294:2542-2545.

Kim I, Saunders TL, Morrison SJ (2007) Sox 17 dependence distinguishes the transcriptional regulation of fetal from adult hematopoietic stem cells. Cell 130:470-483.

Kirstetter P, Anderson K, Porse BT et al (2006) Activation of the canonical Wnt pathway leads to loss of hematopoietic stem cell repopulation and multilineage differentiation block. Nat Immunol 7:1048-1056.

Koch U, Wilson A, Cobas M et al (2008) Simultaneous loss of beta- and gamma-catenin does not perturb hematopoiesis or lymphopoiesis. Blood 111:160-164.

Kollet O, Dar A, Shivtiel S et al (2006) Osteoclasts degrade endosteal components and promote mobilization of hematopoietic progenitor cells. Nat Med 12:657-664.

Kyba M, Perlingeiro RC, Daley GQ (2002) HoxB4 confers definitive lymphoid-myeloid engraftment potential on embryonic stem cell and yolk sac hematopoietic progenitors. Cell 109:29-37. 
Lo Celso C, Fleming HE, Wu JW et al (2009) Live-animal tracking of individual haematopoietic stem/progenitor cells in their niche. Nature 457:92-96.

Lymperi S, Horwood N, Marley S et al (2008) Strontium can increase some osteoblasts without increasing hematopoietic stem cells. Blood 111:1173-1181.

Mancini SJ, Mantei N, Dumortier A et al (2005) Jagged1-dependent Notch signaling is dispensable for hematopoietic stem cell self-renewal and differentiation. Blood 105:2340-2342.

Miyamoto K, Araki KY, Naka K et al (2007) Foxo3a is essential for maintenance of the hematopoietic stem cell pool. Cell Stem Cell 1:101-112.

Naka K, Muraguchi T, Hoshii T et al (2008) Regulation of reactive oxygen species and genomic stability in hematopoietic stem cells. Antioxid Redox Signal 10:1883-1894.

Nakamura Y, Arai F, Iwasaki H et al (2010) Isolation and characterization of endosteal niche cell populations that regulate hematopoietic stem cells. Blood 116:1422-1432.

Naveiras O, Nardi V, Wenzel PL et al (2009) Bone-marrow adipocytes as negative regulators of the haematopoietic microenvironment. Nature 460:259-263.

Nilsson SK, Johnston HM, Whitty GA et al (2005) Osteopontin, a key component of the hematopoietic stem cell niche and regulator of primitive hematopoietic progenitor cells. Blood 106:1232-1239.

Orford KW, Scadden DT (2008) Deconstructing stem cell self-renewal: genetic insights into cellcycle regulation. Nat Rev Genet 9:115-128.

Orkin SH, Zon LI (2008) Hematopoiesis: an evolving paradigm for stem cell biology. Cell 132:631-644.

Owusu-Ansah E, Banerjee U (2009) Reactive oxygen species prime Drosophila haematopoietic progenitors for differentiation. Nature 461:537-541.

Papayannopoulou T, Craddock C, Nakamoto B et al (1995) The VLA4/VCAM-1 adhesion pathway defines contrasting mechanisms of lodgement of transplanted murine hemopoietic progenitors between bone marrow and spleen. Proc Natl Acad Sci U S A 92:9647-9651.

Park IK, Qian D, Kiel M et al (2003) Bmi-1 is required for maintenance of adult self-renewing haematopoietic stem cells. Nature 423:302-305.

Petit I, Szyper-Kravitz M, Nagler A et al (2002) G-CSF induces stem cell mobilization by decreasing bone marrow SDF-1 and up-regulating CXCR4. Nat Immunol 3:687-694.

Qian H, Buza-Vidas N, Hyland CD et al (2007) Critical role of thrombopoietin in maintaining adult quiescent hematopoietic stem cells. Cell Stem Cell 1:671-684.

Qiao XT, Ziel JW, McKimpson W et al (2007) Prospective identification of a multilineage progenitor in murine stomach epithelium. Gastroenterology 133:1989-1998.

Rafii S, Lyden D, Benezra R et al (2002) Vascular and haematopoietic stem cells: novel targets for anti-angiogenesis therapy? Nat Rev Cancer 2:826-835.

Reya T, Duncan AW, Ailles L et al (2003) A role for Wnt signalling in self-renewal of haematopoietic stem cells. Nature 423:409-414.

Rieger MA, Hoppe PS, Smejkal BM et al (2009) Hematopoietic cytokines can instruct lineage choice. Science 325:217-218.

Sacchetti B, Funari A, Michienzi S et al (2007) Self-renewing osteoprogenitors in bone marrow sinusoids can organize a hematopoietic microenvironment. Cell 131:324-336.

Sato T, Vries RG, Snippert HJ et al (2009a) Single Lgr5 stem cells build crypt-villus structures in vitro without a mesenchymal niche. Nature 459:262-265.

Sato T, Onai N, Yoshihara $\mathrm{H}$ et al (2009b) Interferon regulatory factor-2 protects quiescent hematopoietic stem cells from type I interferon-dependent exhaustion. Nat Med 15:696-700.

Scheller M, Huelsken J, Rosenbauer F et al (2006) Hematopoietic stem cell and multilineage defects generated by constitutive beta-catenin activation. Nat Immunol 7:1037-1047.

Schofield R (1978) The relationship between the spleen colony-forming cell and the haemopoietic stem cell. Blood Cells 4:7-25.

Song X, Wong MD, Kawase E et al (2004) Bmp signals from niche cells directly repress transcription of a differentiation-promoting gene, bag of marbles, in germline stem cells in the Drosophila ovary. Development 131:1353-1364.

Stier S, Ko Y, Forkert R et al (2005) Osteopontin is a hematopoietic stem cell niche component that negatively regulates stem cell pool size. J Exp Med 201:1781-1791. 
Suda T, Suda J, Ogawa M (1984) Disparate differentiation in mouse hemopoietic colonies derived from paired progenitors. Proc Natl Acad Sci U S A 81:2520-2524.

Sugiyama T, Kohara H, Noda M et al (2006) Maintenance of the hematopoietic stem cell pool by CXCL12-CXCR4 chemokine signaling in bone marrow stromal cell niches. Immunity 25:977-988.

Taichman RS (2005) Blood and bone: two tissues whose fates are intertwined to create the hematopoietic stem-cell niche. Blood 105:2631-2639.

Takakura N, Huang XL, Naruse T et al (1998) Critical role of the TIE2 endothelial cell receptor in the development of definitive hematopoiesis. Immunity 9:677-686.

Till JE, McCulloch EA (1961) A direct measurement of the radiation sensitivity of normal mouse bone marrow cells. Radiat Res 14:213-222.

Tothova Z, Kollipara R, Huntly BJ et al (2007) FoxOs are critical mediators of hematopoietic stem cell resistance to physiologic oxidative stress. Cell 128:325-339.

Tulina N, Matunis E (2001) Control of stem cell self-renewal in Drosophila spermatogenesis by JAK-STAT signaling. Science 294:2546-2549.

Tumbar T, Guasch G, Greco V et al (2004) Defining the epithelial stem cell niche in skin. Science 303:359-363.

Visnjic D, Kalajzic Z, Rowe DW et al (2004) Hematopoiesis is severely altered in mice with an induced osteoblast deficiency. Blood 103:3258-3264.

Walkley CR, Olsen GH, Dworkin S et al (2007) A microenvironment-induced myeloproliferative syndrome caused by retinoic acid receptor gamma deficiency. Cell 129:1097-1110.

Whetton AD, Graham GJ (1999) Homing and mobilization in the stem cell niche. Trends Cell Biol 9:233-238.

Wilson A, Trumpp A (2006) Bone-marrow haematopoietic-stem-cell niches. Nat Rev Immunol 6:93-106.

Wilson A, Laurenti E, Oser G et al (2008) Hematopoietic stem cells reversibly switch from dormancy to self-renewal during homeostasis and repair. Cell 135:1118-1129.

Xie T, Spradling AC (2000) A niche maintaining germ line stem cells in the Drosophila ovary. Science 290:328-330.

Xie Y, Yin T, Wiegraebe W et al (2009) Detection of functional haematopoietic stem cell niche using real-time imaging. Nature 457:97-101.

Yalcin S, Zhang X, Luciano JP et al (2008) Foxo3 is essential for the regulation of ataxia telangiectasia mutated and oxidative stress-mediated homeostasis of hematopoietic stem cells. J Biol Chem 283:25692-25705.

Yamashita YM, Jones DL, Fuller MT (2003) Orientation of asymmetric stem cell division by the APC tumor suppressor and centrosome. Science 301:1547-1550.

Yilmaz OH, Valdez R, Theisen BK et al (2006) Pten dependence distinguishes haematopoietic stem cells from leukaemia-initiating cells. Nature 441:475-482.

Yoshihara H, Arai F, Hosokawa K et al (2007) Thrombopoietin/MPL signaling regulates hematopoietic stem cell quiescence and interaction with the osteoblastic niche. Cell Stem Cell 1:685-697.

Zhang J, Li L (2008) Stem cell niche: microenvironment and beyond. J Biol Chem 283:9499-9503.

Zhang J, Niu C, Ye L et al (2003) Identification of the haematopoietic stem cell niche and control of the niche size. Nature 425:836-841.

Zhang J, Grindley JC, Yin T et al (2006) PTEN maintains haematopoietic stem cells and acts in lineage choice and leukaemia prevention. Nature 441:518-522. 